\title{
Applications of Wireless Sensor Network in the Field of Medical and Healthcare
}

\author{
Chunmei Wang ${ }^{1, \text { a }}$, Tianyu $\mathrm{Cao}^{2}$, Tianlei $\mathrm{Gao}^{1}$, Zhaoyang Liu ${ }^{1}$ \\ ${ }^{1}$ Shandong Provincial Key Laboratory of Computer Networks, Shandong Computer Science Center \\ (National Supercomputer Center in Jinan), Jinan, 250014, China \\ ${ }^{2}$ School of Mathematical Sciences, Dalian University of Technology, Dalian, 116024, China \\ aemail: wangcmscsc@sina.com
}

Keywords: WSN; System structure; Medical application

\begin{abstract}
Wireless sensor network (WSN) is an emerging technology which integrated sensor technology, embedded computing technology, modern network, wireless communication technology, distributed information processing technology. This technology has wide applications. With the development of technology, WSN will be a great influence in many aspects of medical practice. This paper discusses the application of WSN in the medical field.
\end{abstract}

\section{Introduction}

Wireless sensor network (WSN) is a kind of distributed sensor network, whose terminals are the sensors can sense and check the outside world. Sensors of the WSN use wireless way to communicate, therefore the network settings are flexible and the equipment location can change at any time. Then the multiple sensors form a multi-hops self-organizing network through wireless communication.

The development of WSN thanks to the rapid development of Micro-company-Mechanism System (MEMS), System on Chip (SoC), wireless communications and low-power embedded technology. WSN widely used in military, intelligent transportation, environmental monitoring, healthcare and so on.

WSN applications in the healthcare field is called Wireless body area network (WBAN). WBAN is one important technical of personal health information collection and transmission, and has important practical significance and industrial prospects, attracts widespread attention in industry, academia and standardization organizations.

Wireless body area network (WBAN), is wearable wireless network computing devices [1][2][3][4]. WBAN devices are mobile devices can be embedded in the human body, installed on a fixed position on the surface of the body, or carried by humans to different places [5].

The development trend of WBAN is equipment miniaturization. The network is composed of several small body sensor units (BSUs) together with a single body central unit (BCU) [6][7]. WBAN technology development has began from 1995, using the wireless personal area network (WPAN) technology to realize communications in, on or around the human body. About six years later, the word "BAN" began to specifically indicate that the system communication is completely close to the human body [8]. A WBAN systems can use WPAN wireless technology as a gateway to achieve longer distances. Through the gateway device, the body's wearable devices can be connected to the Internet. As a result, medical professionals can obtain the patient's data online using the Internet no longer depending on the patient's position [9].

Through the WBAN, people can communicate or synchronize data with their personal electronic devices such as PDA (Personal Digital Assistant). Through WBAN people can also communicate with other networks such as others WBAN, wired or wireless access networks or mobile communication network, and become a part of the whole communication network. WBAN can also communicate to any terminal such as a PC (personal computer), mobile phone, telephone, media playback devices, digital cameras, game consoles, etc, shown as Figure 1. WBANs make the body to be part of the communication network, and the wearable computing, ubiquitous computing will 
also with the popularity of WBAN applications become the basic characteristics of people's daily life.

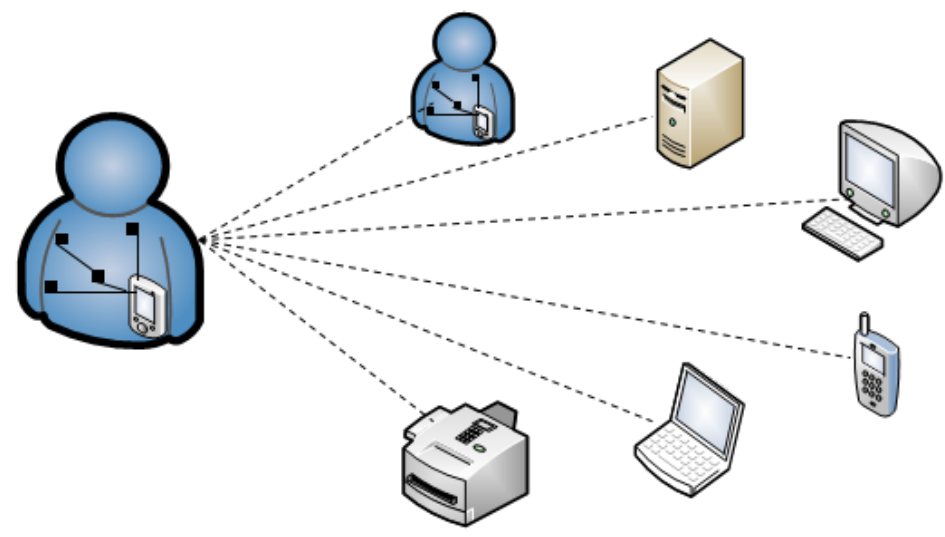

Fig.1. WBAN communicates with other networks or devices

\section{The System Structure of WBAN}

Wireless body area networks uses the mode of distributed acquisition or perception, and then centralized processing. From an architectural point of view, a complete WBAN usually contains three layers, as shown in Figure 2.

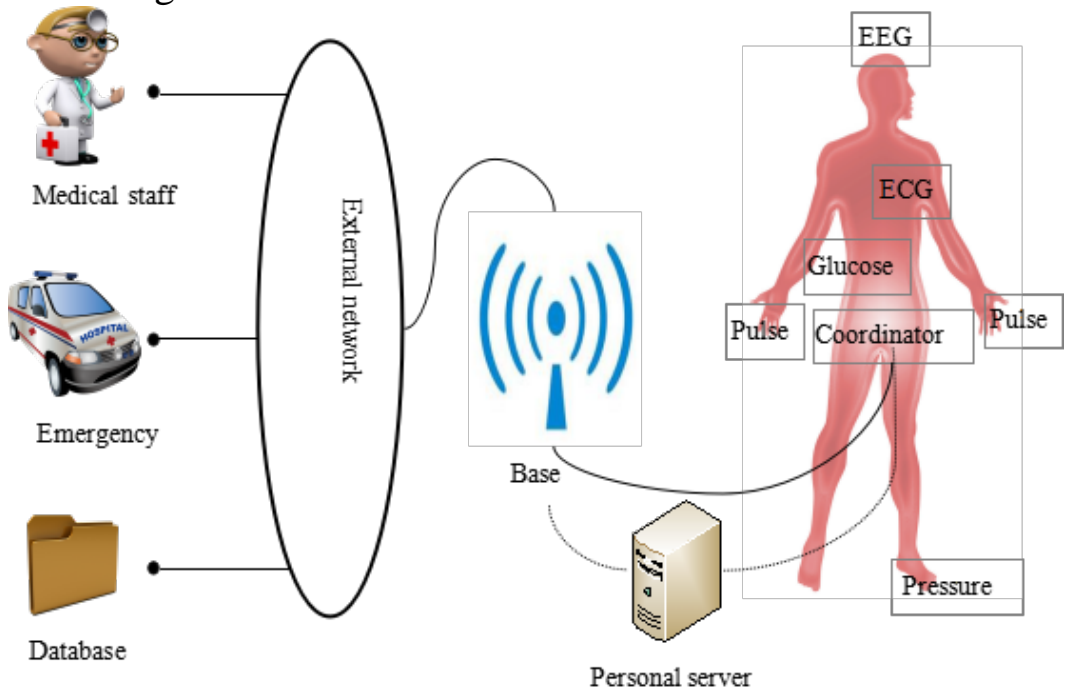

Fig.2. The system structure of WBAN

The first layer includes a set of sensor nodes or equipment, which have detection function, can monitor all kinds of human physiological parameters or human motion (e.g., ECG sensors, EEG sensor, blood pressure sensor, etc.). These nodes are usually designed simply, mainly used for collecting or monitoring physiological data of the body and environment, to deal with relevant information and then send to the network coordinator or sink node. The second layer is a wearable or family own personal data terminals, where data from nodes will be transferred to. Then personal server accepts data from coordinator or sink node through wireless or wired network, temporarily store data to the back-end database, and simply analyze and integrate. This layer is also connected with the external network. The base station in figure 2 is used for GSM (Global System for Mobile Communication)/GPRS (General Packet Radio Service)/3G Communication. The third layer provides a variety of applications, usually owned by hospitals and other medical institutions, is responsible for monitoring the personal data terminal in a fixed area, to analyze, judge and store their message, and remind medical staff to make the correct medical treatment in time. The patient or physician can view their relevant medical information anytime and anywhere using the Internet to access to the backstage database. The above three layers transmit data through wireless channel and involve a variety of protocol. 
A wireless body area network contains one or more nodes, as shown in Figure 3.

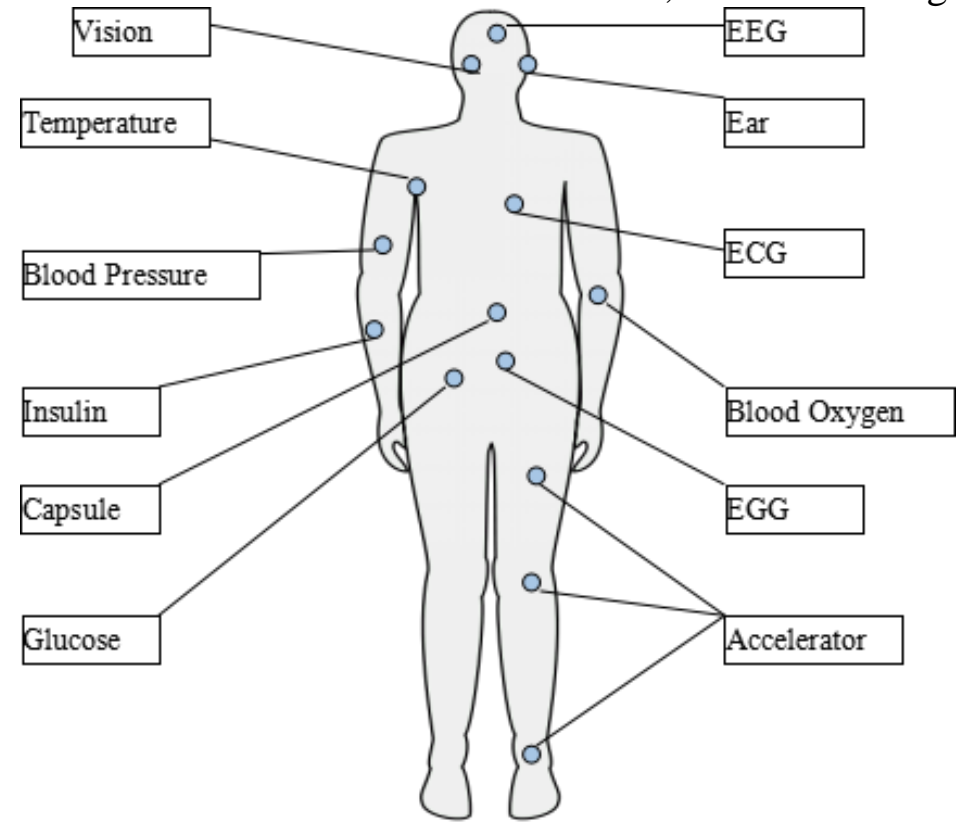

Fig.3. The coordinator and other common WBAN sensor nodes

The basic structure of WBAN sensor nodes including processing module, sensor module, wireless module and power module. Sensor module is used for external sensor signal perception, acquisition and conversion. The WBAN sensors are used for living organisms, especially the human body which is the most complex system in the world, the physiological variables exist high correlation. Therefore WBAN sensors need to extract physical quantity from various physiological phenomena, and get reliable and meaningful measurement data. At the same time, it need to ensure the safety of the tested object.

Wireless module is for data communication between nodes. The commonly used wireless communication technology are: IEEE802.1lb, IEEE802.15.4 (ZigBee), Bluetooth, UWB, RFID, IrDA, etc. Monitoring system mostly uses IEEE802.15.4 (ZigBee), bluetooth to realize node and data communication between nodes and base stations.

Power module is used to provide energy for the node, which is the basic module of the wireless sensor nodes. However, the energy of sensor node is limited since the node is restricted by volume. Hence, in the node design, it need to take a series of effective methods to save energy, which should consider low power consumption and high precision. In addition, WBAN sensor nodes could not replace battery frequently, which will affect the person's normal life. Therefore, the WBAN nodes should have a longer life cycle.

\section{The Applications of Wireless Body Area Network}

From the point of the resent research, the main applications of WBAN scenario has the following: (1) the human body sensor networks, (2) the human body (health) condition monitoring, (3) wearable media player, (4) video streaming applications, (5) the remote control.

Healthcare and medical is the most typical application of wireless body area networks, including the human body sensor network and human health monitoring, etc. Human body sensor is mainly to collect some important data information of the human body, such as heart rate, EEG, ECG, body temperature, blood pressure, and the level of certain chemicals, such as oxygen in the blood and drug data transmitted to the control center. The control center analyze these data, and further determine physical condition of the monitored human body. Current studies suggest that the human body or internal can be deployed about 10-20 various types of sensors. In the form of wireless to monitor patient, the patient can get rid of cable equipment, can bring great convenience to the hospital and patients. To monitor patients in hospital generally uses the star topology, the data information transferred to the patient monitor. When the abnormal situation occurs, this monitor can 
provide an alarm. In addition, the topology used outside of the hospital or home to monitor the patient or the elderly is the same, however the difference is: the monitor replaced by a data collector or gateway, such as PDA, mobile phone, etc. Thus, in the human body to build a mini wireless body area networks, the patient can monitor their own health, and the health information can be transmitted to remote monitoring center in time through the existing cellular network/WIFI. This shows, the wireless body area networks is part of the ubiquitous healthcare network, through wide-area air interface to connect to the back-end infrastructure, medical and health institutions, which gives patients greater freedom space, and improve the efficiency and real-time monitoring.

(1) Wearable WBAN: wearable health monitoring system, allowing the close monitoring of vital signs change of the individual, and provide feedback, to help maintain the best state of health. WBAN wearable medical applications, can be further divided into: the disability assistance and the human management.

In the aspect of disability assistance, WBAN have a large number of applications. For example, using WBAN to provide help to people who have visual impairment. The camera attaches on the sunglasses and crutches, taking real-time photos to the signal processor, then the signal processor explains these photos, to convert information like objects or roadmap into sound signals to the user. Also WBAN brings benefits to difficult speaker. The sensors between fingers and hands can detect the movement of the fingers and hands, at the same time, it can detect the relative position between fingers and hand, WBAN can collect these data, and sends the data real-time to signal processor, the finger language can be interpreted to sound, so as to effectively solve the problem.

In the aspect of human management, WBAN can provide real-time health monitoring. For example, glucose mobile phones is a glucose monitoring module, can be used in patients with diabetes. The phone receives glucose diagnostic information from glucose module, which can then be stored and sent to the doctor for analysis [4].

(2) Implantable WBAN: This kind of application is about the node implanted under the skin, or in the blood vessels. Cancer detection aspects, for example, cancer mortality estimates increase 50\% by 2020, reaches 15 million. The implanted WBAN sensors that monitor the cells within the human body, will allow doctors to continuous diagnosis of tumor, do not need to provide a biopsy and the analysis of processing is real time [4].

Medical equipment remote control: WBAN systems integrate into a remote medical system, can remind the medical staff life-threatening information, and to deal with timely. By the innovation application of wireless body area networks, long-term recording the various physiological signals and activities can improve healthcare and life quality. The Ambient Assisted Living (AAL) is the most famous one, in which each WBAN and back-end medical network communicate in wireless way. The purpose of ALL is to assist families to prolong the patient's self care, minimize the critical patients' dependence on personal care, improve the quality of life, and reduce social cost. In fact, AAL will promote the characteristics of a new generation of IT systems, such as the expected behavior, context awareness, user friendly and flexibility [4]. A typical WBAN application scenario is shown in Figure 4. 


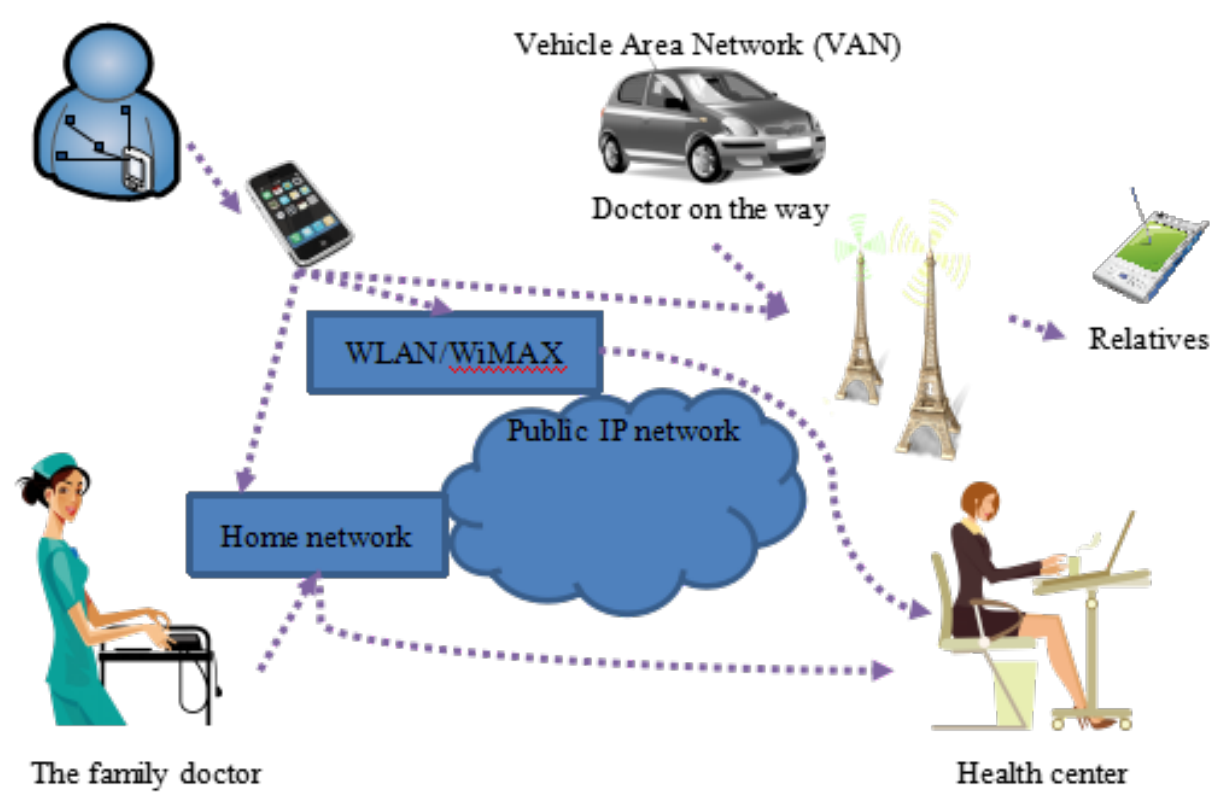

Fig.4. A WBAN application scenario

Applications in the field of non-medical are largely concentrated in the entertainment industry. Entertainment and games are the attractive field, WBAN take an important role in this field, including synchronization of video and audio, video or audio stream and video game controller, etc., these applications have similarity with wearable computer. WBAN can not only bring convenience to users, but also provide feasibility for users to share the game. Shared audio or video resource is one of the advantages to the traditional wireless technology compared with WBAN.

\section{Conclusion}

This article mainly states the applications of wireless sensor network in the field of medical and wearable, analyze the architecture of WBAN and its applications in many aspect. Medical advances, the extension of human life, and people' attention to their health, promote the development of electronic health solutions based on the wireless personal area network and body area network. In recent years, there the many researches and applications about WBAN, however, for the moment, WBAN technology is still immature. In the future, there are a lot of work needs to be done in terms of standardization and industrialization for WBAN.

\section{References}

[1] Body area networks[EB/OL]. http://www.ieee802.org/15/pub/TG6.html.

[2] Sana Ullah, Henry Higgins, Bart Braem, Benoit Latre, Chris Blondia, Ingrid Moerman, Shahnaz Saleem, Ziaur Rahman, and Kyung Sup Kwak. A comprehensive survey of wireless body area networks[J]. Journal of medical systems, 2012 36(3) 1065-1094.

[3] Min Chen, Sergio Gonzalez, Athanasios Vasilakos, Huasong Cao, and Victor C. M. Leung. Body area networks: A survey[J]. Mobile Networks and Applications, 2011 16(2) 171-193.

[4] Samaneh Movassaghi, Mehran Abolhasan, Justin Lipman, David Smith, and Abbas Jamalipour. Wireless Body Area Networks: A Survey[J]. IEEE Communications Surveys and Tutorials, 2014 16(3) 1658-1686.

[5] Stefan Poslad. Ubiquitous computing smart devices, smart environments and smart interaction[J]. Sirirajmedj Com, 2009 67(10) 642-644.

[6] Robert Schmidt, Thomas Norgall, Joachim Mörsdorf, Josef Bernhard, and Thomas von der Grün. Body Area Network BAN--a key infrastructure element for patient-centered medical applications[J]. 
Biomedizinische Technik, 2002 47(s1a) 365-368.

[7] Tony O'Donovan, John O'Donoghue, Cormac J. Sreenan, David Sammon, Philip O'Reilly, and Kieran A. O'Connor. A context aware wireless body area network (BAN). Pervasive Computing Technologies for Healthcare, 2009 [C]. IEEE, 2009: 1-8.

[8] Mehmet R. Yuce. Implementation of wireless body area networks for healthcare systems[J]. Sensors and Actuators A: Physical, 2010 162(1) 116-129.

[9] Mehmet R. Yuce and Jamil Khan. Wireless body area networks: technology, implementation, and applications[M]. Pan Stanford Publishing, 2011. 